J. Lake Sci. (湖泊科学), 2013, 25(2):283-288

http: //www. jlakes.org. E-mail : jlakes@niglas.ac.cn

(C) 2013 by Journal of Lake Sciences

\title{
太湖蓝藻水样中藻蓝蛋白提取方法比较
}

\author{
张 静 ${ }^{1}$, 韦玉春 ${ }^{1 * *}$, 王国祥 ${ }^{2}$,杨 $飞^{2}$, 程春梅 ${ }^{1}$, 夏晓瑞 ${ }^{1}$ \\ (1: 南京师范大学虚拟地理环境教育部重点实验室,南京 210046) \\ (2: 江苏省环境演变与生态建设重点实验室,南京 210046)
}

摘 要: 以 2011 年 8 月 20 日采集的太湖梅梁湾的夏季蓝藻水华为研究对象, 通过 12 个样点平行样的藻蓝蛋白实验提 取, 基于光谱吸收特征和浓度值, 对反复冻融法、超声波法、溶胀法、丙酮法的提取效果进行比较评价. 结果表明:4 种方法 获取的藻蓝蛋白提取液在 $620 \mathrm{~nm}$ 附近出现吸收峰, 其中, 反复冻融法的峰高最强, 超声波法最弱, 说明反复冻融法的提取 效果优于其他方法; 反复冻融法、超声波法、溶胀法获取的部分蓝蛋白提取液在 $670 \mathrm{~nm}$ 附近具有次吸收峰, 与藻蓝蛋白标 样的吸收曲线存在差异; 反复冻融法、超声波法提取的藻蓝蛋白浓度值变异系数小于 0.6 , 表明这两种方法较其他方法稳 定; 反复冻融法提取的藻蓝蛋白浓度值高于其他 3 种方法, 可推荐作为太湖蓝藻水样中藻蓝蛋白的提取方法.

关键词: 藻蓝蛋白; 提取方法; 太湖;梅梁湾;蓝藻水华;分光光度法

\section{Comparison of extraction methods for phycocyanin from cyanobacterica blooms water samples in Lake Taihu}

\author{
ZHANG Jing ${ }^{1}$, WEI Yuchun ${ }^{1}$, WANG Guoxiang ${ }^{2}$, YANG Fei $^{2}$, CHENG Chunmei ${ }^{1} \&$ XIA Xiaorui ${ }^{1}$ \\ (1: Key Lab of Virtual Geographic Environment, Ministry of Education, Nanjing Normal University, Nanjing 210046, P. R. \\ China) \\ (2: Jiangsu Key Laboratory of Environmental Change and Ecological Construction, Nanjing 210046, P. R. China)
}

\begin{abstract}
Taking cyanobacterica blooms water samples collected from 12 different sites in Meiliang Bay of Lake Taihu in August 20,2011 as a research object, the phycocyanin extraction method including freeze thawing cycle, ultrasonic wave extracting, cellmelting and acetone were compared based on absorption spectrum and phycocyanin concentration. Results showed that the absorption spectrum of phycocyanin extracted by these methods have significant peak at $620 \mathrm{~nm}$. The peak height is quite different among these methods: the highest peak is observed in freeze thawing cycle while the lowest in ultrasonic wave extracting, indicating that the four methods can extract phycocyanin from water samples, and freeze thawing cycle method is superior to others; Some phycocyanin specturm obtained by freeze thawing cycle, ultrasonic wave extracting and cell-melting appeared the second peak near 670 $\mathrm{nm}$, and differentiated with the specturm of phycocyanin standard sample; The variation coefficient of phycocyanin concentration in the freeze thawing cycle method and ultrasonic wave method are less than 0.6, showing that their result is more precise and stable; The phycocyanin concentration showed that extraction efficiency of freeze thawing cycle method is better than the others, and so is recommended as a common method for phycocyanin extraction from cyanobacterica blooms water samples in Lake Taihu.
\end{abstract}

Keywords: Phycocyanin; extraction methods; Lake Taihu; Meiliang Bay; cyanobacterica blooms; spectrophotometry

蓝藻是一类极其古老、微小的原核生物, 具有极强的生态竞争优势. 伴随着富营养化, 蓝藻会大量生长 与繁殖, 形成水华, 给水体水质带来严重的危害 ${ }^{[1]}$. 藻蓝蛋白 (phycocyanin, PC) 在 $620 \mathrm{~nm}$ 附近有一个吸收 峰, 是蓝藻的特征色素蛋白之一 ${ }^{[2]}$. 在环境监测中, 特别是在蓝藻水华频发的水域, 藻蓝蛋白浓度能有效地

* 国家自然科学基金项目 (40771152)、江苏省普通高校自然科学研究计划项目 (09KJA420001) 和江苏高校优势学科 建设工程项目 (1411109012) 联合资助. 2012-04-23 收稿;2012-09-03 收修改稿. 张静, 女, 1986 年生, 硕士研 究生;E-mail:jingzhang537@ 126. com.

** 通信作者;E-mail: weiyuchun@ njnu. edu. cn. 
表征蓝藻生物量. 准确提取水体中的藻蓝蛋白,对藻蓝蛋白浓度定量、蓝藻水华监测具有重要意义.

目前, 实验使用的藻蓝蛋白的提取方法较多, 常用的有反复冻融法、化学试剂处理法、溶胀法、超声波法 等 ${ }^{[3-21]}$. 典型研究如 Padgett 等 ${ }^{[4]}$ 使用反复冻融法提取了铜绿微囊藻中的藻蓝蛋白; 曲文娟等 ${ }^{[14]}$ 采用脉冲超 声辅助技术提取钝顶螺旋藻中的藻蓝蛋白; 朱丽萍等 ${ }^{[15]}$ 以溶胀法提取多管藻中的藻蓝蛋白. 但是, 不同研究 侧重点不同, 基于藻种不同, 得到的结论存在差异. 对于藻蓝蛋白提取效果的评价, 上述研究也主要基于提 取液的藻蓝蛋白浓度结果进行, 较少做吸收光谱方面的分析研究, 而吸收光谱是藻蓝蛋白浓度定量的基 础 ${ }^{[22]}$. 此外, 已有的藻蓝蛋白提取研究多侧重于实验室内培养的藻类. 太湖是富营养化严重的浑浊水体, 湖 泊中藻种多样, 藻种结构也具有明显的时空差异 ${ }^{[23]}$. 因此, 在使用上述方法提取太湖水体中的藻蓝蛋白时需 要做具体分析.

本文以 2011 年 8 月 20 日采集的太湖梅梁湾蓝藻水华样品为研究对象, 选取反复冻融法、超声波法、溶 胀法、丙酮法提取水样中的藻蓝蛋白. 基于光谱吸收特征和藻蓝蛋白浓度统计结果对各种提取方法进行评 价, 以期为太湖蓝藻水华的监测提供基础支撑.

\section{1 材料与方法}

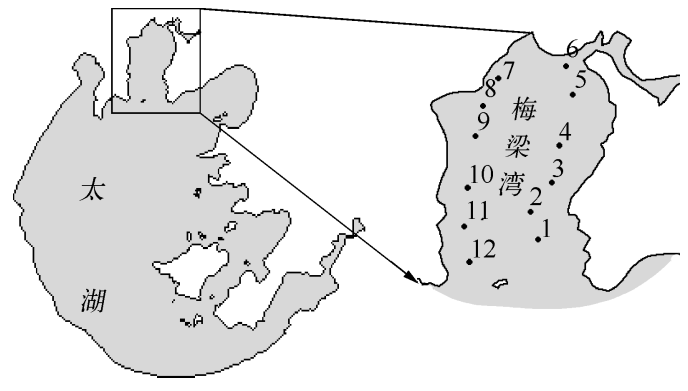

图 1 太湖梅梁湾采样点分布

Fig. 1 Distribution of sampling sites in Meiliang Bay of Lake Taihu

\section{1 样品采集}

水样采自太湖梅梁湾湖区表层 $30 \mathrm{~cm}$ 水体,共 12 个样点 (图 1). 采集日期为 2011 年 8 月 20 日. 采样 期间天气晴朗, 风速为 $0.8 \sim 2.2 \mathrm{~m} / \mathrm{s}$. 水样采集后放 人冷藏箱内运回实验室进行分析.

藻蓝蛋白标准样品购自美国 Sigma 公司, 编号 为 P6161.

\section{2 样品准备}

将野外采集的太湖水华溶液混匀, 每种藻蓝蛋白 的提取方法分别平行采集相同体积的太湖样品, 采用 $1.2 \mu \mathrm{m}$ 孔径的 Whatman GF/C 滤膜真空原抽滤, 不 同样点采集的水样水质状况不同, 过滤体积稍有差 异. 以 $1^{\#} \sim 12^{\#}$ 表示各样点水样.

\section{3 太湖蓝藻水样中藻蓝蛋白的提取}

分别参照下述方法提取 $1^{\#} \sim 12^{\#}$ 太湖蓝藻水样中的藻蓝蛋白. 每种提取方法设置 3 个平行样.

反复冻融法 ${ }^{[4]}$ : 将附着藻体的滤膜放人冻存管中, 同时加人 $0.05 \mathrm{~mol} / \mathrm{L}$ 磷酸盐缓冲液于 $-20^{\circ} \mathrm{C}$ 冰箱冷 冻, 然后室温避光解冻, 如此反复冻融 7 次. 解冻的样品以 12000 转 $/ \mathrm{min}$ 离心 $10 \mathrm{~min}$, 取离心后的上清液作 为待测液.

超声波法 ${ }^{[14]}$ : 将附着藻体的滤膜置于超声波仪中, 以 $0.05 \mathrm{~mol} / \mathrm{L}$ 的磷酸盐缓冲液为溶媒, 超声波处理 $20 \min ($ 功率 $800 \mathrm{~W}$ ). 超声波处理结束后以 12000 转 $/ \min$ 离心 $10 \mathrm{~min}$, 取离心后的上清液作为待测液.

溶胀法 ${ }^{[15]}$ : 将附着藻体的滤膜放人烧杯中, 同时加人 $0.05 \mathrm{~mol} / \mathrm{L}$ 磷酸盐缓冲液, 在 $15^{\circ} \mathrm{C}$ 的条件下溶胀 提取, 溶胀时间为 $3 \mathrm{~d}$. 提取结束后以 12000 转 $/ \mathrm{min}$ 离心 $10 \mathrm{~min}$, 取离心后的上清液作为待测液.

丙酮法 ${ }^{[8]}$ : 将附着藻体的滤膜放人试管中, 同时加人 $90 \%$ 的丙酮抽提脂溶性色素, 以 12000 转 $/ \mathrm{min}$ 离心 $10 \mathrm{~min}$. 取上清液作为丙酮法的对比待测液, 同时将剩余的蓝色沉淀加一定量 $0.05 \mathrm{~mol} / \mathrm{L}$ 磷酸盐缓冲液, 置 于磁力振荡搅拌器上高速搅拌 $20 \mathrm{~min}$, 再以 12000 转 $/ \mathrm{min}$ 离心 $10 \mathrm{~min}$, 取离心后的上清液作为丙酮法的待 测液.

\section{4 标准样品配置}

使用 $0.05 \mathrm{~mol} / \mathrm{L}$ 的磷酸盐缓冲液, 配置浓度为 $20 、 10 、 7 、 5 、 4 、 2 、 1$ 和 $0.5 \mathrm{~mol} / \mathrm{L}$ 的藻蓝蛋白标准样品系 列,使用 P1 至 P8 表示.

\section{5 藻蓝蛋白浓度测定}

使用岛津 UV-2450/UV-2550 分光光度计测量各待测液和标准样品的吸光度. 分光光度计扫描波长为 
$350 \sim 750 \mathrm{~nm}$, 分辨率为 $1 \mathrm{~nm}$.

根据 Bennett 等 $^{[3]}$ 的公式求取藻蓝蛋白浓度:

$$
C_{\mathrm{PC}}=\left(A_{615}-0.474 A_{652}\right) / 5.34
$$

式中, 藻蓝蛋白浓度 $C_{\mathrm{PC}}$ 的单位为 $\mathrm{mg} / \mathrm{ml} ; A_{615} 、 A_{652}$ 分别是藻蓝蛋白在 $615 、 652 \mathrm{~nm}$ 处的吸光度.

\section{6 数据处理}

数据分析在 SPSS 16.0 软件中进行.

\section{2 结果与分析}

\section{1 不同方法提取的藻蓝蛋白吸收光谱结果}

反复冻融法、超声波法、溶胀法、丙酮法提取的各样品的藻蓝蛋白吸收光谱如图 2, 图中各样品的吸光 度值以其平行样的吸光度均值表示.
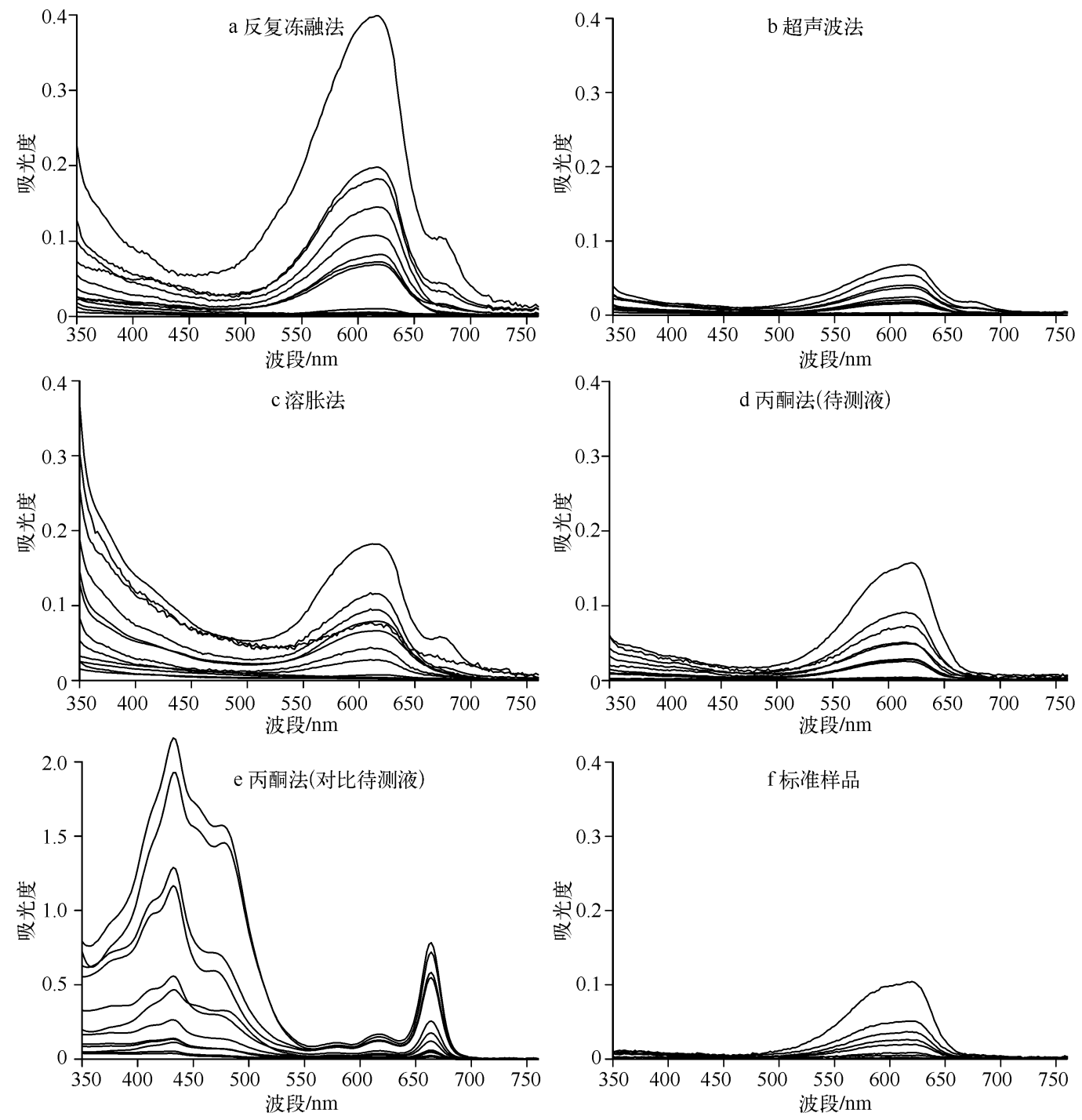

图 2 不同方法提取的藻蓝蛋白吸收光谱

Fig. 2 Absorption spectrums of phycocyanin extracted by different methods 
在 350 750 nm 的测量波长范围内, 丙酮法藻蓝蛋白提取液 (图 2d) 的吸收光谱形状和吸收峰的位置与 标准样品 (图 2f) 一致. 对比测量的丙酮上清液 (图 2e) 在波长 430、480、620 和 $670 \mathrm{~nm}$ 处均具有吸收峰, 且 $430 、 480$ 和 $670 \mathrm{~nm}$ 处吸收峰的峰高比 $620 \mathrm{~nm}$ 强. 反复冻融法 (图 2a) 、超声波法 (图 2b)、溶胀法 (图 2c) 的 藻蓝蛋白提取液在 $620 \mathrm{~nm}$ 附近出现主吸收峰, 其中, 反复冻融法 $620 \mathrm{~nm}$ 的峰高最强, 超声波法最弱. 此外, 反复冻融法、超声波法、溶胀法获取的部分水样的藻蓝蛋白提取液在 $670 \mathrm{~nm}$ 附近还具有次吸收峰, 其位于 $650 \sim 700 \mathrm{~nm}$ 区间的谱型特征与标准样品存在差异.

表 1 样品中藻蓝蛋白含量的变异系数

Tab. 1 Variation coefficients of phycocyanin concentration in different samples

\begin{tabular}{ccccc}
\hline 样点 & $\begin{array}{c}\text { 反复 } \\
\text { 冻融法 }\end{array}$ & $\begin{array}{c}\text { 超声 } \\
\text { 波法 }\end{array}$ & 溶胀法 & 丙酮法 \\
\hline $1^{\#}$ & 0.51 & 0.34 & 0.38 & 0.43 \\
$2^{\#}$ & 0.28 & 0.41 & 0.31 & 0.66 \\
$3^{\#}$ & 0.18 & 0.03 & 0.35 & 0.56 \\
$4^{\#}$ & 0.42 & 0.57 & 0.65 & 0.41 \\
$5^{\#}$ & 0.03 & 0.25 & 0.49 & 0.70 \\
$6^{\#}$ & 0.32 & 0.51 & 0.73 & 0.64 \\
$7^{\#}$ & 0.31 & 0.11 & 0.19 & 0.36 \\
$8^{\#}$ & 0.56 & 0.52 & 0.64 & 0.73 \\
$9^{\#}$ & 0.06 & 0.39 & 0.26 & 0.08 \\
$10^{\#}$ & 0.25 & 0.09 & 0.07 & 0.28 \\
$11^{\#}$ & 0.17 & 0.20 & 0.32 & 0.39 \\
$12^{\#}$ & 0.05 & 0.13 & 0.25 & 0.37 \\
\hline
\end{tabular}

\section{2 不同方法提取的藻蓝蛋白浓度结果}

以 Bennett 等的公式计算各提取方法对应的 $1^{\#} \sim 12^{\#}$ 水样的藻蓝蛋白浓度值. 由于不同点位的 水质状况不同, 水样的平行样的藻蓝蛋白浓度均 值具有差异. 因而, 文中使用变异系数 (样品平行 样的浓度值标准差与均值的比值) 衡量不同方法 提取的藻蓝蛋白的差异性. 变异系数如表 1 , 平行 样的均值结果见图 3. 反复冻融法、超声波法不 同水样各个平行样的变异系数均低于 0.6 (表 $1)$, 表明这两种方法平行样的结果具有较好的 一致性, 测量操作误差较小. 由不同方法提取的 藻蓝蛋白浓度比较结果 (图 3 ) 可知, 反复冻融 法提取的 $1^{\#} \sim 12^{\#}$ 太湖水样的浓度值均高于其 他 3 种方法, 溶胀法次之, 超声波法的提取效果 最不理想.

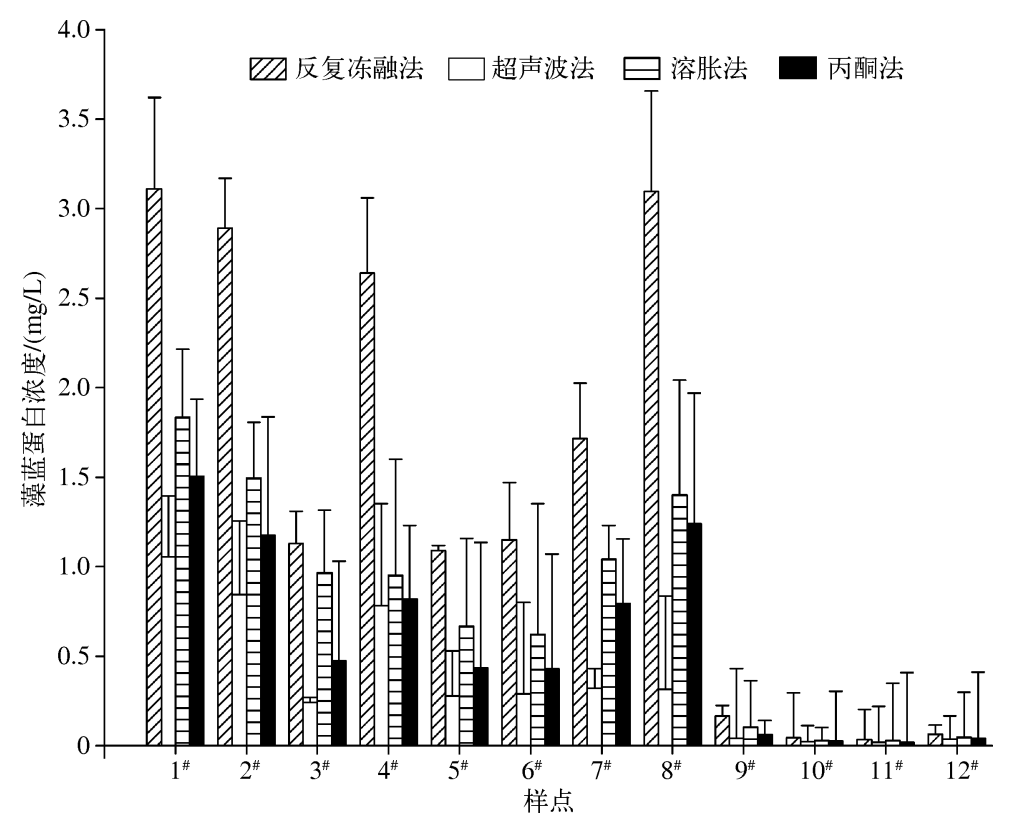

图 3 不同方法提取的藻蓝蛋白浓度比较结果 ( T6 和 T8 号太湖水样的藻蓝 蛋白浓度较其他样点高, 为便于作图比较, 这两个样点的浓度值分别取 其实际浓度值的 $1 / 10$ 和 $1 / 20$ 表示)

Fig. 3 Comparasion of the phycocyanin concentration under different extraction methods 


\section{3 讨论}

每种物质都有自身的特征吸收光谱, 吸收峰波长可以对已知物质定性 ${ }^{[22]}$. 已有研究结果 ${ }^{[3-21]}$ 表明, 藻蓝 蛋白吸收峰位于 $620 \mathrm{~nm}$ 附近. 本文中反复冻融法、超声波法、溶胀法、丙酮法的藻蓝蛋白提取液在 $620 \mathrm{~nm}$ 附 近具有吸收峰, 反复冻融法 $620 \mathrm{~nm}$ 的峰高最强, 超声波法最弱. 这说明 4 种方法均能不同程度地提取出太湖 蓝藻水华样品中的藻蓝蛋白, 其中, 反复冻融法的提取效果优于其他方法. 反复冻融法 (图 2a)、超声波法 (图 2b)、溶胀法 (图 2c) 获取的部分水样的藻蓝蛋白提取液在 $670 \mathrm{~nm}$ 附近还具有吸收作用, $650 \sim 700 \mathrm{~nm}$ 区 间的谱型特征与标准样品 (图 2f) 存在差异. 这主要是由于反复冻融法、超声波法、溶胀法获取的藻蓝蛋白提

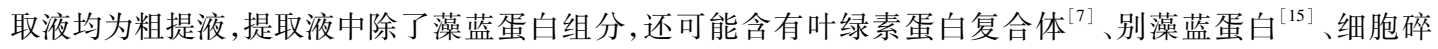
片 $^{[17]}$ 等物质组分. 这些物质组分的存在会使得藻蓝蛋白提取液的吸收光谱特征发生改变. 余九九等 ${ }^{[7]}$ 的研 究结果表明, 在以液氮冻融、超声波破碎、蒸馏水低温自溶、氯化䥻自溶 4 种方法提取出极大螺旋藻藻泥、藻 粉中的藻蓝蛋白的同时, 镶嵌于类囊体膜上的叶绿素蛋白复合体也会随之脱落共存于提取液中, 并对其 $670 \mathrm{~nm}$ 附近的吸收光谱特性产生影响. 本文中反复冻融法、超声波法、溶胀法获取的部分水样的藻蓝蛋白提 取液在 $670 \mathrm{~nm}$ 附近出现吸收峰, 说明用这 3 种方法提取太湖蓝藻水华样品中的藻蓝蛋白时受到了叶绿素蛋 白复合体的影响. 此外, 与反复冻融法、超声波法、溶胀法, 以及余九九等 ${ }^{[7]}$ 研究中的蒸馏水低温自溶、氯化 钙自溶等方法不同, 丙酮法先以丙酮作为浸提溶剂破坏藻体细胞结构. 丙酮为有机溶剂, 能使脂溶性的叶绿 素 a 从复合体上脱落下来且以色素的形式溶于其中 ${ }^{[18]}$. 故而, 在丙酮上清液弃除、磷酸盐缓冲液再悬浮后获 取的藻蓝蛋白提取液的吸收光谱上, 未出现叶绿素蛋白复合体位于 $670 \mathrm{~nm}$ 附近 ${ }^{[24-25]}$ 的吸收峰(图 2d).

以往的研究 ${ }^{[12,14]}$ 已证实了超声波法能有效地破碎藻类细胞, 提取出样品中的藻蓝蛋白. 但是本实验中, 无论是从图谱分析还是计算浓度值的统计结果来看, 超声波法的提取效果均不理想, 提取不完全现象严重. 究其原因, 主要受研究对象的影响. 与室内培养的纯藻种样品不同, 野外采集太湖水样中的藻体多以包裹体 形式存在 ${ }^{[26]}$. 包裹体表面附有群体胶鞘 ${ }^{[27]}$, 破壁所需的超声强度较室内藻种大. 此外, 朱丽萍等 ${ }^{[15]}$ 和尹兴 娟等 ${ }^{[18]}$ 的研究表明, 藻蓝蛋白热稳定性差, 在 $40^{\circ} \mathrm{C}$ 即发生变性. 增加超声波作用的强度和延长作用的时间 均会导致热效应增加, 使得细胞内蛋白等有效组分的破坏. 因此, 在太湖蓝藻水样的藻蓝蛋白提取中, 单纯 用超声波来破碎藻体细胞结构是不够的.

相较于超声波法, 溶胀法在 $620 \mathrm{~nm}$ 藻蓝蛋白特征峰处的峰值和相应的计算浓度值均有所提高, 但其提 取率仍低于反复冻融法. 谭啸等 ${ }^{[23]}$ 的研究表明太湖水体中藻种众多, 藻种结构具有明显的时空差异. 余九九 等 ${ }^{[7]}$ 、韦萍等 ${ }^{[9]}$ 的研究证实不同藻种所需溶胀溶剂不同. 因此, 多藻种共存可能是溶胀法提取效果不理想的 一个影响因素. 从吸收光谱图 (图 2) 来看, 溶胀法在 $350 \sim 500 \mathrm{~nm}$ 光谱区间的吸光度值衰减率高于其他方 法. 溶胀法提取周期长, 提取过程中藻体一直与液态溶剂接触, 导致藻体细胞结构破碎, 产生的降解物增多, 这可能是产生该差异的一个重要原因. 此外, 大量有机降解物的产生也会改变溶液的 $\mathrm{pH}$ 环境, 使得藻蓝蛋 白活性降低 ${ }^{[10]}$, 反而不利于藻蓝蛋白的提取.

丙酮法的藻蓝蛋白提取液在 $670 \mathrm{~nm}$ 附近未出现叶绿素蛋白复合体的吸收峰, 但其在 $620 \mathrm{~nm}$ 处的峰高 低于反复冻融法和溶胀法. 尹兴娟等 ${ }^{[18]}$ 的研究表明, 藻蓝蛋白由载体蛋白和藻蓝素 (发色团) 组成. 细胞结 构破碎后溶出的水溶性藻蓝蛋白可溶于氯仿等有机溶剂中, 变为脂溶性的藻蓝素. 实验中使用的丙酮为有 机溶剂, 能使脂溶性的色素溶于其中. 因此, 本文同时测量了丙酮法提取的丙酮上清液 (图 2e) 和再溶解后的 蓝色沉淀部分 (图 2d), 并对其吸收光谱和浓度结果进行了比较. 吸收光谱图中 (图 2), 两种待测液均在 $620 \mathrm{~nm}$ 附近出现吸收峰, 且二者的吸收峰强度相当, 说明丙酮法能提取出样品中的部分藻蓝蛋白, 亦说明丙 酮方法提取出的藻蓝蛋白会以藻蓝素的形式溶于丙酮而存在不同程度的损失. 浓度统计结果 (图 3) 中丙酮 法提取的藻蓝蛋白浓度值小于反复冻融法和溶胀法也证明了这一点. 此外, 在丙酮法提取中, 上清液和蓝色 沉淀的分离存在不确定性, 蓝色沉淀部分再溶解也存在不完全现象, 均会对其提取测量的稳定性产生影响.

吸收光谱的图谱分析结果 (图 2) 和藻蓝蛋白浓度计算结果 (图 3) 均表明, 反复冻融法的提取效果明显 优于其他方法. 反复冻融法主要通过细胞内冰粒的形成和剩余细胞液盐浓度的增高引起溶胀, 使细胞壁、细 胞膜破碎, 藻胆体内的藻蓝蛋白溶出. 李冰等 ${ }^{[12]}$ 的研究指出, 藻蓝蛋白在低温状态下具有较好地稳定性. 因 此, 当样品中存在包裹体且藻类组成不同时, 可以通过增加反复冻融次数和降低冻融温度的方式来提高提 取率. 此外, 藻体在反复冻融提取的大部分时间里均处于结冻状态, 细胞结构破碎降解产生有机质的含量较 
溶胀法小, $\mathrm{pH}$ 环境稳定, 易于保持藻蓝蛋白的活性. 但是, 与超声波法、溶胀法类似, 在细胞结构破碎藻蓝蛋 白溶出的同时, 位于类囊体膜上的叶绿素蛋白复合体也同时释放出来. 从吸收光谱图 (图 2) 来看, 叶绿素蛋 白复合体位于 $670 \mathrm{~nm}$ 附近的吸收峰对 $650 \mathrm{~nm}$ 处的吸光度产生了影响. 考虑到常用的藻蓝蛋白浓度计算公 式 $^{[3,5]}$ 中定义的 $650 \mathrm{~nm}$ 处吸光度为别藻蓝蛋白的吸收, 因此, 在以后的研究工作中, 还需要开展更多的实验 对比工作来分析叶绿素蛋白复合体对太湖水样中藻蓝蛋白提取的影响.

综上所述, 反复冻融法的提取效果明显优于其他方法, 可推荐作为一种太湖蓝藻水样中藻蓝蛋白的实 验室提取方法.

致谢: 太湖水样采集得到了南京师范大学地理科学学院研究生王否、孙晓鹏、周宇的帮助, 藻蓝蛋白的提取 实验得到了南京师范大学生命科学学院李建宏教授的指导以及研究生夏明升的帮助, 在此表示衰心感谢.

\section{4 参考文献}

［1］王扬才, 陆开宏. 蓝藻水华的危害及治理动态. 水产学杂志, 2004, 17 (1) : 90-93.

[ 2 ] Jupp DL, Kirk JTO, Harris GP. Detection, identification and mapping of cyanobacteria using remote sensing to measure the optical quality of turbid inland waters. Australian Journal of Freshwater Research, 1994, 45(5) : 801-828.

[ 3 ] Bennett A, Bogorad L. Complementary chromatic adaptation in a filamentous blue-green alga. The Journal of Cell Biol, 1973, 58 (2) : 419-435.

[ 4 ] Padgett MP, Krogmann DW. Large scale preparation of pure phycobiliproteins. Photosynthesis Research, 1987, 11(3): 225-235.

[ 5 ] 高洪峰. 不同生长期坛紫菜中藻胆蛋白的含量变化. 海洋与湖沼, 1993, 24(6) : 645-648.

[ 6 ] 翟文川, 潘红秃. 太湖蓝藻中天然色素的分离提取及测定. 湖泊科学, 1997, 9(3) : 284-285.

[ 7 ] 余九九, 李宽钰, 吴庆余. 螺旋藻的藻胆蛋白提取及稳定性研究. 海洋通报, 1997, 16 (4) : 26-28.

[ 8 ] 何 佳, 赵启美, 田 娟. 螺旋藻藻蓝素稳定性的研究. 生物学杂志, 1998, 15(5): 10-17.

[ 9 ] 韦 萍, 李 环, 张成武. 极大螺旋藻藻蓝蛋白的提取与纯化. 南京化工大学学报, 1999, 21 (3) : 62-65.

[10] 邹 宁, 孙东红, 焦爱霞等. 鱼腥藻藻蓝蛋白的提取. 水产养殖, 2005, 26(1) : 5-7.

[11] Badrish S, Beena K, Ujjval T et al. Extraction, purification and characterization of phycocyanin from Oscillatoria quadripunctulata - isolated from the rocky shores of Bet-Dwarka, Gujarat, India. Process Biochemistry, 2006, 41(9): 2017-2023.

[12] 李 冰, 张学成, 高美华等. 钝顶螺旋藻㩰蓝蛋白提取纯化新工艺. 海洋科学, 2007, 31(8) : 48-52.

[13] Patil G, Raghavarao KSMS. Aqueoustwo phase extraction for purification of c-phycocyanin. Biochemical Engineering Journal, 2007, 34(2) : 156-164.

[14] 曲文娟, 马海乐, 张厚森. 针顶螺旋藻藻蓝蛋白的脉冲超声辅助提取技术. 食品科技, 2007, 13(5): 135-138.

[15] 朱丽萍, 颜世敢, 张玉忠. 溶胀因素对多管藻藻胆蛋白粗提得率和纯度影响. 食品研究与开发, 2009, 30 (9): $65-68$.

[16] 于 光, 马宇翔, 张成武等. 盐泽螺旋藻藻蓝蛋白的分离纯化. 食品与生物技术学报, 2009, 28(4) : 521-524.

[17] 沈 强, 沈银武, 刘永定等. 滇池水华蓝藻藻蓝蛋白的分离纯化与毒性研究. 环境化学, 2009, 28 (4): 497-501.

[18］尹兴娟, 唐建国, 刘继宪. 藻蓝素的提取及其光学性质研究. 应用化工, 2010, 39 (4) : 486-490.

[19] Hemlata GP, Fareha B. Studies on Anabaena sp. NCCU-9 with special reference to phycocyanin. Journal of Algal Biomass Utilization, 2011, 2(1) : 30-51.

[20］廖晓霞, 张学武. 高效分离纯化藻蓝蛋白新法. 食品工业科技, 2011, 32(6) : 273-275.

[21] 张允允, 陈敏. 蓝隐藻藻蓝蛋白的分离、纯化及性质研究. 烟台大学学报: 自然科学与工程版, 2011, 24 (4): 281-286.

[22] 刘志广. 分析化学. 北京: 高等教育出版社, 2008: 227-228.

[23] 谭 啸, 孔繁翔, 曾庆飞. 太湖中微囊藻群落的季节变化分析. 生态与农村环境学报, 2009, 25(1) : 47-52.

[24] French CS, Brown JS, Lawrence MC. Four universal forms of chlorophyll a. Plant Physiol, 1972, 49(3) : 421-429.

[25] 刘 梅, 刘其芳, 张 宪等. 固氮蓝藻 Anabaena sp. 7120 的叶绿素蛋白复合体的分离和光谱特性的研究. 水生生 物学报, $1985,9(1): 1-8$.

[26] Roesler CS, Perry MJ. Modeling in situ phytoplankton absorption from total absorption spectra in productive inland marine waters. Limnology and Oceanography, 1989, 34(8) : 1510-1523.

[27] 孔繁翔, 高 光. 大型浅水富营养化湖泊中蓝㩰水华形成机理的思考. 生态学报, 2005, 25(3): 591-595. 